\title{
Chitosan Based Dressings for Wound Care
}

\author{
Shakeel Ahmed ${ }^{1}$, Mudasir Ahmad', Megha Jayachandran², Mohammad Amir Qureshi' ${ }^{3}$ and Saiqa Ikram ${ }^{1 *}$ \\ ${ }^{1}$ Department of Chemistry, Jamia Millia Islamia, New Delhi-110025, India \\ ${ }^{2}$ Department of Chemistry, Syracuse University, Syracuse, New York-13210, USA \\ ${ }^{3}$ Department of Applied Sciences and Humanities, Jamia Millia Islamia, New Delhi-110025, India
}

\begin{abstract}
Biopolymer researchers have advanced new strategies to improve the properties of wound dressings over the last decade which is becoming one of the most swiftly growing fields in the biomedical and pharmacy. The advantages of these biopolymers (particularly chitosan) is that, they can be easily processed into different forms which needs to be for different biomedical applications. Chitosan is the well-known natural biopolymer which is safe to be use, biocompatible and biodegradable in nature. This review provides an outline of the chitosan properties and its biomedical properties toward applications in anti-inflammatory and wound caring management.
\end{abstract}

Keywords: Chitosan; Anti-inflammatory; Wound healing; Biopolymer; Biomedical

\section{Introduction}

In all biomedical applications today, polymers are playing an important role. Various notable progresses in polymeric materials like composite, blends, nano-polymeric materials, etc. have followed their exploitation [1-5]. The several biopolymers e.g. starch, and other carbohydrate polymers produced by fungi and bacteria and animal derived biopolymers like chitosan, chitin, gelatin, collagen have found to be favorable biopolymers for industrial applications [6].

The basic properties required for a wound dressings are maintenance of moisture and nutrients at site, oxygenation, inflammation control, fibroblast proliferation and epithelialisation. Chitosan is one of such polymers which fulfil such requirement for a wound dressings due to its antimicrobial character and was found to be potent material for future wound dressings. The commercial polysaccharides like dextran, starch, alginic acid, carrageenan and heparin are either acidic or neutral polymers, but chitosan being basic polymer. Chitosan is insoluble in neutral or basic $\mathrm{pH}$, due to its free amino groups, while in acidic $\mathrm{pH}$, due to protonation of amino groups it becomes soluble in water. The solubility of chitosan is dependent on the distribution of free amino and $\mathrm{N}$-acetyl groups [7-9].

\section{Chitosan structure and its origin}

Chitosan is a copolymer which consists of $\beta$ - $(1 \rightarrow 4)$-linked 2-acetamido-2-deoxy-D-glucopyranose and 2-amino-2-deoxyD-glucopyranose units which is generally obtained by alkaline deacetylation of chitin the main component of the exoskeleton of crustaceans.

Chitin is second most abundant biopolymer after cellulose and is the main constituent of the exoskeleton in animals particularly in crustaceans, mollusks and insects. Deacetylation of chitin yields chitosan (when deacetylation $>50 \%$ ), which is produced in several forms such as powder, paste, film and fiber. Chitosan is soluble in dilute acids like acetic acid, lactic acid, malic acid, formic acid and succinic acid [10-13]. In acidic medium, chitosan is cationic in nature and readily interacts with negatively charged molecules such as proteins, alginate, carrageenan, bile acids and phospholipids. Nonetheless, chitosan may also interact with chelate metal ions such as copper, cadmium, iron, mercury and magnesium and help in their removal from water [14].

Due to the biodegradability, biocompatibility, antimicrobial effect and its non-toxicity, much attention has been paid to chitosan for its biomedical applications. Chitosan stimulates hemostasis and accelerate regeneration of tissues, therefore it found to be useful for wound healing management. For a material to be used for biomedical research, a natural product is preferred because these materials are more biocompatible than synthetic materials. Chitosan is an attractive material for a tissue engineering scaffold because it has structural similarities to glycosaminoglycan and is hydrophilic in nature. The monomeric unit of chitosan, $\mathrm{N}$-acetyl glucosamine is important in healing of wound $[15,16]$.

An effective approach is to modify chitosan surface to provide excellent bulk properties for developing clinically applicable chitosan. Chitosan can be fabricated into a stable, porous scaffold via surface modification and lyophilization. However, blending with various additives may affect its biocompatibility. Therefore, evaluation of the biocompatibility of various biomedical-grade chitosan derivatives is necessary to engineer material that is of high quality and biocompatible for human wound management. They offer the advantage of being easily processed into gels, membranes, nanofibers, nanofibrils, beads, microparticles, nanoparticles, scaffolds and sponge-like forms (Figure 1). Due to these properties and its biocompatibility, it has versatile applications in tissue engineering, wound healing, as excipients for drug delivery and gene delivery $[17,18]$. In order to advance the performance of chitosan, crosslinking emerge as another strategy for its improvement. The hydrogel crosslinking properties mainly depend on their crosslinking density, namely the ratio of moles of crosslinking agent to the moles of polymer repeating units. For the formation of a network structure, the number of crosslinks per chain are required. The various structure of chitosan hydrogels formed are: (a) chitosan cross-linked with itself, (b) hybrid polymer network, (c) semi-interpenetrating network, and (d) ionic crosslinking. Depending

*Corresponding author: Saiqa Ikram, Department of Chemistry, Jamia Millia Islamia, New Delhi-110 025, India, Tel: +91-11-26981717 extn. 3255; E-mail: sikram@jmi.ac.in/shakeelchem11@gmail.com

Received October 08, 2015; Accepted October 30, 2015; Published November 06, 2015

Citation: Ahmed S, Ahmad M, Jayachandran M, Qureshi MA, Ikram S et al. (2015) Chitosan Based Dressings for Wound Care. Immunochem Immunopathol 1: 106. doi: 10.4172/2469-9756.1000106

Copyright: (C) 2015 Ahmed S, et al. This is an open-access article distributed under the terms of the Creative Commons Attribution License, which permits unrestricted use, distribution, and reproduction in any medium, provided the original author and source are credited. 


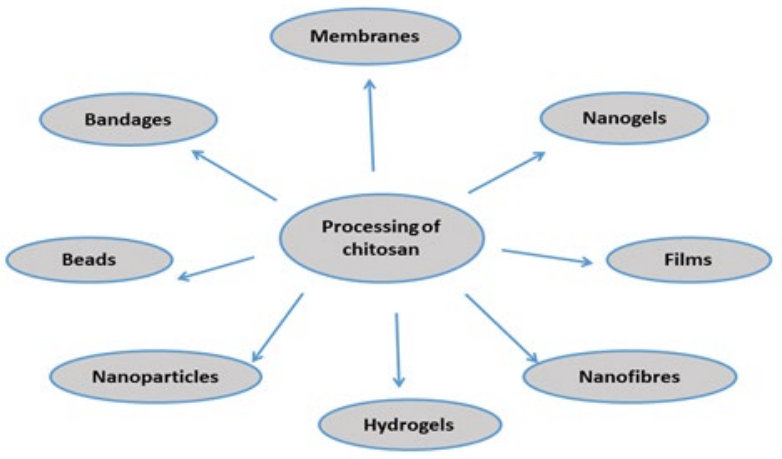

Figure 1: Schematic representation of processing chitosan into different forms.

on the nature of the cross linker, the main interactions forming the network are either covalent or ionic bonds [19-21].

\section{Chitosan in wound care}

Chitosan is a potent antimicrobial agent and its antimicrobial character is due to presence of its cationic nature. An antimicrobial is an agent that kills microorganisms or inhibits their growth [22]. The substance or treatment that reduces inflammation is known as anti-inflammatory. Anti-inflammatory drugs make up about half of analgesics that reduces inflammation thereby remedying pain in contrast to opioids, which affect the central nervous system. Chitosan has a variety of promising biomedical applications and at this time, is considered as a new innovative material in drug delivery systems, wound healing, antibacterial, fat binder, hemostatic agent, hypocholesterolemic effect as indicated by the large number of studies published over the last few years [12]. Due to intraperitoneal administration of acetic acid, chitosan treatment reduces inflammatory pain in a dose-dependent manner. Bradykinin is one of the main substances related to pain. It was reported that the bradykinin concentration during administration of a chitosan-acid acetic solution in the peritoneal lavage fluid was lower than during the administration of a $0.5 \%$ acetic acid solution, suggesting that chitosan has analgesic effects. Chitosan that is formulated for wound management may induce analgesia by providing a cool, pleasant and soothing effect when applied to an open wound. Excellent pain relief was conferred by chitosan when it was applied as a topical agent to open wounds, such as burns, skin abrasions, skin ulcers and skin grafted areas [23].

Chitosan-dependent antimicrobial activity has been observed against various microorganisms, such as fungi, algae and bacteria. These antimicrobial effects are controlled by various intrinsic factors including type of chitosan, the degree of their polymerization, the host, the chemical or nutrient composition of the substrates and many environmental conditions. The photo-cross-linked electrospun mats containing quartinized chitosan were found to be promising materials for wound-dressing [24]. The antimicrobial activities of chitosan differs mainly in live host plants e.g. the fungicidal effects of $\mathrm{N}$-carboxymethyl chitosan are different in vegetable and graminea hosts and is more immediate on fungi and algae than on bacteria [25]. Furthermore, in the presence of more than $0.025 \%$ chitosan, the growth of Escherichia coli, Fusarium, Alternaria and Helminthosporium is inhibited [26]. The inhibition of growth of these micro-organisms is due to cationic amino groups of chitosan which bind to anionic groups. During the infectious period of a burn wound, bacterial infection may delay the healing and probably cause serious complications, such as sepsis. Chitosan that is incorporated with minocycline hydrochloride was developed to achieve both wound healing enhancement and antibacterial effects [27]. Due to anti-inflammatory effects of chitosan, these are beneficial for the treatment of prolonged inflammation at the wound site. Watersoluble chitosan significantly suppresses the secretion and expression of proinflammatory cytokines (e.g., tumor necrosis factor- $\alpha$ and interleukin-6) and inducible nitric oxide synthase in astrocytes, the predominant neuroglial cells in the central nervous system, and is actively involved in cytokine-mediated inflammatory events [28]. Moreover, $\mathrm{N}$-acetylglucosamine is an anti-inflammatory drug and is synthesized in the human body from glucose. It is incorporated into glycosaminoglycans and glycoproteins. Chito-oligosaccharides, which have a molecular weight of $5 \mathrm{kDa}$, showed better anti-inflammatory agents than nonsteroidal anti-inflammatory drug, indomethacin [29]. Chitosan exerts anti-inflammatory effects by inhibiting prostaglandin $\mathrm{E} 2$ and cyclooxygenase- 2 protein expression and attenuating the proinflammatory cytokines (e.g., tumor necrosis factor- $\alpha$ and interleukin$1 \beta)$. However, chitosan treatment increases the expression of the antiinflammatory cytokine, interleukin-10 [30].

In various disciplines of health care and hygienic applications, chitosan is used for contact disinfectants in many biomedical applications, including wound dressing, drug delivery carrier, tissue engineering, gene delivery, haemodialysis, bio-imaging, etc. (Figure 2) [31-33].

Wound healing is a particular biological route allied to the general phenomenon of growth and tissue regeneration. It progresses through a series of inter-reliant and corresponding stages in which a variety of cellular and matrix components act together to regenerate the integrity of damaged tissue and replacement of lost tissue [34]. It is a complex and dynamic regenerative process that is divided into four phases: haemostasis, inflammation, proliferation, and remodelling [35]. Chitosan hydrogel coated grafts which were crosslinked upon irradiation with UV-light, exhibited resistance against $E$. coli in vitro and in vivo. E. coli is a gram negative bacteria mostly present on site of wounds. This hydrogel directly acted as antibacterial agent on a Dacron graft, and was effective in inhibiting the local infection [36]. A number of studies showed that chitosan showed wound healing properties. The biocompatible carboxyethyl chitosan/poly(vinyl alcohol) nanofibers were prepared by electrospinning of aqueous solution as wound dressing material. Cell culture results showed that these fibrous mats were good in promoting the L929 cell attachment and proliferation

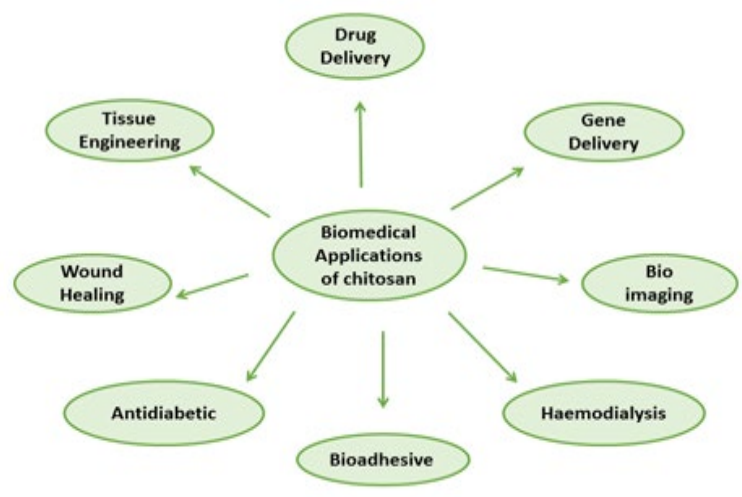

Figure 2: Biomedical applications of chitosan. 
[37]. The wound dressing should be non-allergenic and non-toxic, keep moist environment, allows gas exchanges, absorb wound exudates and protect wound against microbial growth.

Haemorrhage and infectious complications remains the leading cause of early death after trauma and a continuous challenge for caregivers. Chitosan dressings have been developed to address these problems, but these are not so much effective in killing bacteria or controlling bleeding at wound site. The Immobilized poly $(\mathrm{N}$ isopropylacrylamide)/polypropylene nonwoven composites surface crosslinked with glutaraldehyde on chitosan hydrogels displayed good antibacterial ability against $E$. coli and $S$. aureus. The composite was easily stripped-off without damaging newly regenerated tissue at wound site [38,39]. Composite sponge of curcumin/chitosan/gelatin was prepared at the various ratios of chitosan and gelatin showing improved water uptake ability, antibacterial activity, and wound closure. The higher content of gelatin in composite sponge exhibited a faster release behaviour up to $240 \mathrm{~min}$. These composite sponges were also found to enhance the formation of collagen and wound closure in vivo and therefore improved the wound healing activity [40].

Mei Dai et al. reported a biodegradable sponge which is composed of chitosan and sodium alginate loaded with curcumin. Histological results of three different kinds of wound dressings applied on the dorsal skin wound of SD rat. The gauze-treated wounds showed immature granulation tissue with congested vessels and numerous inflammatory cells. The dermis is still under the process of remodelling on $12^{\text {th }}$ day of dressing. However, the granulation tissue and collagen alignment were more advanced in comparison to the gauze-treated wounds in C2A 2 curcumin sponge and $\mathrm{C} 2 \mathrm{~A} 2$ sponge treated wounds. The wounds were under a better soothing conditions revealing well aligned and compact in these two types of wound treatment (Figure 3)[41].

C2A2 sponge was used as wound dressing and full thickness wounds were made on back of each rat (Figure 4 ). The cotton gauze was adhered to wound surface on $4^{\text {th }}$ day of wound and its removal results in less tissue at wound surface. The wound was more contractive on the $12^{\text {th }}$ day than on the $4^{\text {th }}$ postoperative day; the healing of both the C2A2 and C2A2-Curcumin sponges treated wound was obviously faster than gauze-treated wound [41].

The selective sulfation at O-2 and/or O-3 of chitosan may generate potent antiretroviral agents that display a much higher inhibitory effect on the infection of AIDS virus [42]. Chitosan incorporated silver sulfadiazine membrane showed control release with controlled water vapour evaporation, cytocompatibility, and adequate swelling ability with prolonged antibacterial activities [43]. Chitosan-alginate composite membranes also with silver sulfadiazine showed excellent tensile strength and adequate elongation at break in another report. At $50 \%$ alginate concentration, silver release was enhanced proposing release kinetics is dependent on alginate [44]. Wang et al. evaluated the wound healing ability of PEC membrane of CS and alginate suspensions and the membrane was found to be nontoxic toward mouse and human fibroblast cells. In vivo evaluation in incisional wounds in rat proved to be faster healing potential of this composite membrane [45]. The sustained release of silver sulfadiazine showed excellent antibacterial activities against Pseudomonas aeruginosa and Staphylococcus aureus in the in-vivo system as well [46].

Pang et al. developed a composite membrane which showed adequate mechanical strength, with excellent antibacterial activity against Escherichia coli and have tremendous hemostatic potential and was cytocompatible toward human skin fibroblast cells [47].
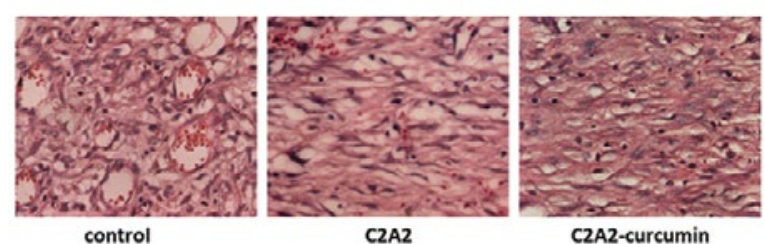

Figure 3: The H\&E stained sections of twelve-day post wounding [41]
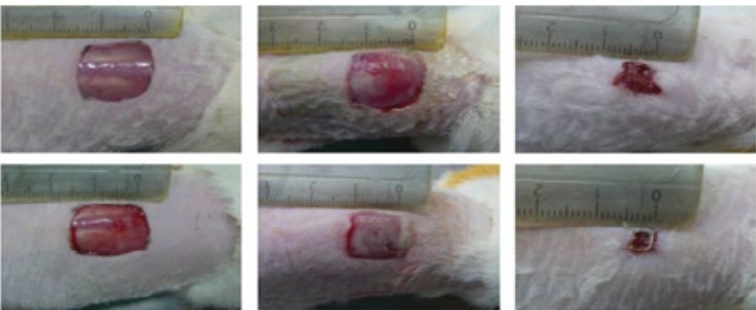

(b)
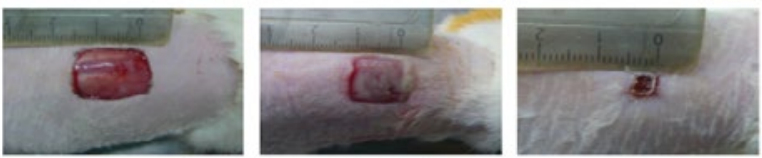

(c)
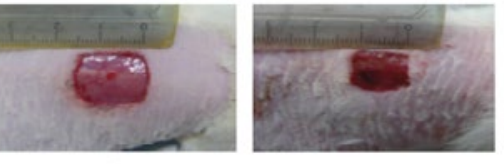

$4^{\text {th }}$ day

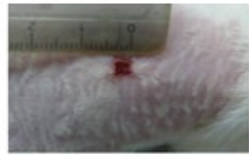

$12^{\text {th }}$ day

Figure 4: Photographs of macroscopic appearance of wound repair covered with (a) cotton gauze as control, (b) C2A2 without curcumin, and (c) C2A2 with curcumin, at day 0 , day 4 , and day 12 , respectively. Reproduced from ref. [41]

Interestingly, a thermo-sensitive film was prepared by combining thiolated chitosan, poly(N-isopropyl acrylamide) and ciprofloxacin, so that the film can easily be removed from wound by decreasing the temperature [48].

The silver nanoparticles are found to be potent antimicrobial agents showing improved antimicrobial activities due to their high surface area leading to incorporation of large number of nanoparticles inside the cell membrane of bacteria thereby promoting its efficiency [49]. Silver nanoparticles have elicit significant interest over the past few decades due to their novel and distinct physical, chemical and biological properties. Due to antimicrobial nature of silver nanoparticles, it has potential to heal the wounds [50-52]. The antibacterial potential of nanosilver incorporated and chitosan hydrogel composite bandage the developed scaffolds showed excellent blood clotting ability, high swelling ratio and controlled biodegradation with excellent antibacterial activities against $S$. aureus and E. coli and were found to be suitable for wound healing [53,54]. The castor oil-based films reinforced with different amounts of CS-modified $\mathrm{ZnO}$ nanoparticles were successfully prepared via solution mixing and casting technique. The nanocomposites exhibited antimicrobial activity against both Gramnegative and Gram-positive bacteria in the presence and the absence of UV light, and the effect increased with the CS- $\mathrm{ZnO}$ concentration. From the morphology, water absorption, thermal stability, biodegradability, cytocompatibility, barrier, mechanical, viscoelastic, wound healing, and antibacterial studies of the these bionanocomposites, these are found to be promising for use in biomedical applications, particularly as wound healing materials [55].

Hydrogel films are very soft and cause no disturbance during its applications in wound dressings. The extracts derived from plants are more potent natural healers and heals the wounds in a natural way. The plant extract is a natural antimicrobial agent which showed low 
compatibility in polymeric hydrogel solution and their efficiency and release in them are found to be low in comparison to their pure form. Hydrogel film encapsulated with gentamycin sulphate, Salix alba and Juglena regia extract showed antimicrobial activities in both disc diffusion method and MIC. Since these properties are the most important properties for ideal wound healing materials which provides a future site to treat wound care system [56-59].

A variety of bacteria are present on wounds which cause infection at wound. This contamination of wound with bacteria encouraged the researchers to develop the wound dressings with improved antimicrobial effects through the incorporation of or not of antimicrobial agents. The therapeutic use of aloe vera, or other material could be helpful in creation of active wound dressing material. The chitosan/aloe-vera based membranes were developed as wound dressing materials. The addition of aloe vera to chitosan enhanced the antimicrobial potential of the resulting membranes. CAV1 (CS:AV:1:1, v/w) showed the highest antibacterial potential compared with the other formulations due to greater content of AV in the membrane, indicating the greater effectiveness of CAV1 as a bactericide which consequently increases its inhibitory potency. The hDFs exhibited a round morphology with cell agglomeration throughout the study period on chitosan membrane. Cells seeded on the CAV membrane showed better spreading and a higher number of cells attached to the surface. The cells were uniformly distributed on the surface of the CAV membrane from days 1 to 7 , exhibiting a spindle-like shape typical of fibroblasts, suggesting good adherence to this surface. Moreover, on $7^{\text {th }}$ day hDFs were well spread on and were able to adhere to both sides of the CAV membrane surface. Despite the good cell adhesion and proliferation observed on the CAV membrane, the higher AV content of CAV1 did not result in the expected increase in cell spreading and proliferation. The chitosan/aloe vera based membranes might be promising wound dressing materials [60].

Chitin grafted poly(acrylic acid) was prepared with the aim of obtaining a hydrogel suitable for wound dressing application. The morphology and behaviour of the cells on the chitin-PAA film were found to be normal after 14 days of culture [61]. Ribeiro et al. isolated fibroblast cells from rat skin and were used to assess the cytotoxicity of the hydrogel. Chitosan hydrogel was able to promote cell adhesion and proliferation and hydrogel and its degradation by-products are non-cytotoxic. This study suggested that chitosan hydrogel may aid the reinstatement of skin architecture [62]. The biocompatible chitosan/ polyethylene glycol diacrylate blend films and their cytotoxicity assessment with mouse fibroblasts (L929) indicated that the material are non-cytotoxic toward growth of L929 cell and had good in vitro biocompatibility and have the potential to be used as wound dressing material [63]. Porous microspheres of chitosan and its derivatives were prepared in order to deliver antigens in a controlled way [64]. Mucoadhesivity of chitosan and its cationic derivatives proved to enhance the adsorption of drugs especially at neutral $\mathrm{pH}$. $\mathrm{N}$-trimethyl chitosan chloride interacts with the negatively charged cell membranes thus, inhibit the growth of microorganisms. Heterogeneous chemical modification of the chitosan film can tune the surface properties of the film; for instance, when a stearoyl group was attached to the chitosan films, they became more hydrophobic and promoted proteins adsorption. When chitosan films were reacted with succinic anhydride or phthalic anhydride, it resulted in more hydrophilic films which promoted lysozyme adsorption $[65,66]$. The PVA/ bentonite, PVA/Ag nanoparticles, PVA/clove extract, and PVA/cellulose nanocomposite hydrogel was reported by Gonzalez et al. PVA/clove hydrogels did not exhibit a homogenous aspect, while PVA/bentonite and PVA/Ag nanocomposite hydrogels showed significant antimicrobial activity against growth of E. coli, good water vapour transmission rate, and adequate water absorbing capacity due to addition of clay and $\mathrm{Ag}$ nanoparticles as filler [67]. For effective wound healing accelerator, water-soluble chitosan/heparin complex was prepared by Kweon et al. using water-soluble chitosan with wound healing ability and heparin with ability to attract or bind growth factor related to wound healing process [68].

\section{Conclusions}

Due to the inherent antimicrobial activity, biocompatibility and biodegradability of chitosan, its find its uses in various biomedical fields. This review summarizes the biomedical applications of chitosan based materials especially wound healing and its antiinflammatory effect. The properties of chitosan such as antibacterial activity, biocompatibility, ability to bind with growth factors and to be processed into a variety forms, makes it an appropriate candidate as scaffold material for wound dressing. Effective dressings should have characteristics optimized for particular type of wound at a reasonable low cost and with minimum inconvenience to patients. Therefore, there is need to manipulate the physical properties of identified systems to achieve such objectives. There may be many chitosan based dressings which need to explore required for effective wound healing. We expect that this article will provide visions on the usage of these important chitosan based materials in the field of biomedical and pharmaceutical sciences.

\section{Acknowledgements}

The author, Shakeel Ahmed gratefully acknowledges the financial support from University Grant Commission (UGC), New Delhi in the form of Senior Research Fellowship.

\section{References}

1. Udmale V, Mishra D, Gadhave R, Pinjare D, Yamgar R (2013) Development Trends in Conductive Nano-Composites for Radiation Shielding. Orienta journal of chemistry 29: 927-936.

2. Mathewa M, Ninanb KN, Thomas S (1998) Compatibility studies of polymerpolymer systems by viscometric techniques: nitrile-rubber-based polymer blends. Polymer 3925: 6235-6241.

3. Leathers TD, Steinbuchel A, Rhee SK (2005) Polysaccharides and Polyamides in the Food Industry. Properties, Production, and Patents. Vol. 1. Polysaccharides, Chapter 11. Wiley-VCH: Weinheim. 387-421.

4. Chee KK (1990) Determination of polymer- polymer miscibility by viscometry European Polymer Journal 26: 423-426.

5. Sun Z, Wang W, Feng Z (1992) Criterion of polymer-polymer miscibility determined by viscometer. European Polymer Journal 28: 1259-1261.

6. Akhilesh VS (2011) Biopolymers in Drug Delivery: A Review. Pharmacologyonline 1: 666-674

7. Sannan T, Kurita K, Iwakura Y (1977) Studies on Chitin. V. Kinetics of Deacetylation Reaction. Polymer journal 9: 649-651.

8. Sannan T, Kurita K, Iwakura Y (1977) Studies on chitin, 2. Effect of deacetylation on solubility. Makromolecular Chemistry 177: 3589-3600.

9. Zhang J, Cao H, Li S, Zhao Y, Wang W, et al. (2015) Characterization of a new family 75 chitosanase from Aspergillus sp. W-2. International Journal of Biological Macromolecules 81: 362-369.

10. Dimzon IKD, Knepper TP (2015) Degree of deacetylation of chitosan by infrared spectroscopy and partial least squares. International Journal of Biological Macromolecules 72: 939-945.

11. Ahmed S, Ahmad M, Ikram S (2014) Chitosan: A Natural Antimicrobial Agent-A Review. Journal of Applicable Chemistry 3: 493-503.

12. Ahmed S, Ikram S (2015) Chitosan \& its derivatives: A review in recent innovations, International Journal of pharmaceutical Sciences and Research 6: 14-30. 
Citation: Ahmed S, Ahmad M, Jayachandran M, Qureshi MA, Ikram S et al. (2015) Chitosan Based Dressings for Wound Care. Immunochem Immunopathol 1: 106. doi: 10.4172/2469-9756.1000106

13. Ahmad M, Ahmed S Swami BL, Ikram S (2015) Preparation and Characterization of Antibacterial Thiosemicarbazide Chitosan as Efficien $\mathrm{Cu}$ (II) Adsorbent. Carbohydrate Polymers. doi: 10.1016/j.carbpol.2015.06.034

14. Ahmad M, Ahmed S, Swami BL, Ikram S (2015) Adsorption of Heavy meta ions: Role of chitosan and cellulose for water treatment. International Journal of pharmacognosy 2: 280-289

15. Muzzarelli RAA (1996) Chitosan-based dietary foods. Carbohydrate Polymers 29: 309-316.

16. Keong LC, Halim AS (2009) In Vitro models in biocompatibility assessment for biomedical-grade chitosan derivatives in wound management. International Journal of Molecular Sciences 10: 1300-1313.

17. Anithaa A, Sowmyaa S, Sudheesh KPT, Deepthi S, Chennazhi KP, et al. (2014) Chitin and chitosan in selected biomedical applications. Progress in Polymer Science 39: 1644-1667.

18. Dina Raafat D, Sahl HG (2009) Chitosan and its antimicrobial potential - a critical literature survey. Microbial Biotechnology 2: 186-201.

19. Sorlier P, Denuziere A, Viton C, Domard A (2001) Relation between the degree of acetylation and the electrostatic properties of chitin and chitosan. Biomacromolecule 2: 765-772.

20. Kamoun EA, Xin Chen X, Eldin MSM, Kenawy ES (2015) Crosslinked poly(viny alcohol) hydrogels for wound dressing applications: A review of remarkably blended polymers. Arabian Journal of Chemistry 8: 1-14.

21. Berger J, Reist M, Mayer JM, Felt O, Peppas NA, et. al (2004) Structure and interactions in covalently and ionically crosslinked chitosan hydrogels for biomedical applications. European Journal of pharmaceutics and biopharmaceutics 57: 35-52.

22. Salmawi KM (2007) Gamma Radiation $\square$ Induced Crosslinked PVA/Chitosan Blends for Wound Dressing. Journal of Macromolecular Science A 44: 541-545.

23. Okamoto Y, Miyatake K, Morimoto M, Shigmasa M, Minami S (2002) Analgesic effects of chitin and chitosan. Carbohydrate Polymer 49: 249-252.

24. Jayakumar R, Prabaharan M, Nair SV, Tamura H (2010) Novel chitin and chitosan nanofibers in biomedical applications. Biotechnology Advances 28: 142-150.

25. Onshima Y, Nishino K, Yonekura Y, Kishimoto S, Wakabayashi S (1987) Clinical application of chitin non-woven fabric as wound dressing. European Journal of Plastic Surgery 10: 66-69.

26. Savard T, Beaulieu C, Boucher I, Champagne CP (2002) Antimicrobial action of hydrolyzed chitosan against spoilage yeasts and lactic acid bacteria of fermented vegetables. Journal of Food Protection 65: 828-833.

27. Joshua S, Boateng kerr $\mathrm{H}$, Matthews howard NE, Stevens gillian ME (2008) Wound healing dressings and drug delivery systems: a review. Journal of Pharmaceutical Sciences 97: 2892-2923.

28. Aoyagi S, Onishi H, Machida Y (2007) Novel chitosan wound dressing loaded with minocycline for the treatment of severe burn wounds. International Journal of Pharmaceutics 330: 138-145.

29. Kim MS, Sung MJ, Seo SB, Yoo SJ, Lim WK, Kim HM (2002) Water-soluble chitosan inhibits the production of pro- inflammatory cytokine in human astrocytoma cells activated by amyloid beta peptide and interleukin-1beta. Neuroscience Letters 321: 105-109.

30. Spindola H, Fernandes J, De Sousa V, Tavaria F, Pintado M, Malcata X Carvalho JE (2009) Anti-inflammatory effect of chitosan oligomers. New Biotechnology 25: S9.

31. Muzzarelli RAA, Jeuniauk C, Gooday GW (1986) Chitin in Nature and Technology, Plenum Press, New York. Section-VI: 435-507.

32. Sjak-Braek G, Anthonsen T, Sandford P (1992) Advances in Chitin and Chitosan. Edited by C. J. Brine, P. A. Sandford and J. P. Zikakis. Elsevier Science Publishers, London. Polymymer International 31: 404.

33. Bourke SL, Al-Khalili M, Briggs T, Michniak BB, Kohn J, et al. (2003) A photocrosslinked poly(vinyl alcohol) hydrogel growth factor release vehicle for wound healing applications. AAPS Pharm Science 5: 101-111.

34. Neethu N, Muthunarayanan M, In-Kyu P, Tin Wui W, Sabu T, et al. (2015) Natural Polymer/Inorganic Material Based Hybrid Scaffolds for Skin Wound Healing. Polymer Reviews 55: 453-490.
35. Fujita M, Kinoshita M, Ishihara M, Kanatani Y, Morimoto Y, et al. (2004) Inhibition of vascular prosthetic graft infection using a photocrosslinkable chitosan hydrogel. The Journal of Surgical Research 121: 135-140.

36. Zhou Y, Yang D, Chen X, Xu Q, Lu F, et al. (2008) Electrospun Water-soluble carboxyethyl chitosan/poly(vinyl alcohol) nanofibrous membrane as potential wound dressing for skin regeneration. Biomacromolecules 9: 349-354.

37. Croisier F, Jérôme C (2013) Chitosan based biomaterial for tissue engineering European Polymer Journal 49: 780-792.

38. Chen KS, Ku YA, Lee CH, Lin HR, Lin FH, et al. (2005) Immobilization of Chitosan gel with cross-linking reagent on PNIPAAm/gel/PP non-woven composite surface. Material Sciences \& Engineering-C 25: 472-478.

39. Chen SP, Wu GZ, Zeng HY (2005) Preparation of high antimicrobial activity thiourea chitosan-Ag+ complex. Carbohydrate Polymer 60: 33-38.

40. Van CN, Van BN, Ming-Fa H (2013) Curcumin-Loaded Chitosan/Gelatin Composite Sponge for Wound Healing Application. International Journal of Polymer Science. http: //dx.doi.org/10.1155/2013/106570

41. Mei D, Xiu Ling Z, Xu X, XiangYK, XingYL, et al. (2009) Chitosan-Alginate Sponge: Preparation and Application in Curcumin Delivery for Dermal Wound Healing in Rat. Journal of Biomedicine and Biotechnology. doi: $10.1155 / 2009 / 595126$

42. Nishimura S, Kai H, Shinada K, Yoshida T, Tokura S, et al. (1998) Regioselective syntheses of sulfated polysaccharides: specific anti-HIV-1 activity of novel chitin sulfates. Carbohydrate Research 306: 427-433.

43. Fwu LM, Yu BW, Shin SS, Chao AC, Juin YL, et al. (2003) Asymmetric chitosan membranes prepared by dry/wet phase separation: a new type of wound dressing for controlled antibacterial release. Journal of Membrane Science 212: $237-254$

44. Meng X, Tian F, Yang J, He CN, Xing N, et al. (2010) Chitosan and alginate polyelectrolyte complex membranes and their properties for wound dressing application. Journal of Materials Science: Materials in Medicine 21: 1751-1759.

45. Wang LS, Khor E, Wee A, Lim LY (2002) Chitosan-alginate PEC membrane as a wound dressing: assessment of incisional wound healing. Journal of Biomedical Material Research 63: 610-618.

46. Mi FL, Wu YB, Shyu SS, Schoung JY, Huang YB, et al. (2002) Control of wound infections using a bilayer chitosan wound dressing with sustainable antibiotic delivery. Journal of Biomedical Material Research 59: 438-449.

47. Pang HT, Chen XG, Ji QX, Zhong de $Y$ (2008) Preparation and function of composite asymmetric chitosan/CM-chitosan membrane. Journal of Materials Science: Materials in Medicine 19: 1413-1417.

48. Radhakumary C, Antonty M, Sreenivasan K (2011) Drug loaded thermoresponsive and cytocompatible chitosan based hydrogel as a potential wound dressing. Carbohydrate Polymers 83: 705-713.

49. Vimala K, Mohan YM, Sivudu KS, Varaprasad K, Ravindra S, et al. (2010) Fabrication of porous chitosan films impregnated with silver nanoparticles: a facile approach for superior antibacterial application. Colloids Surface B Biointerfaces 76: 248-258.

50. Ahmed S, Ikram S (2015) Silver nanoparticles: One Pot Green Synthesis Using Terminalia arjuna Extract for Biological Application. Journal of Nanomedicine and Nanotechnology 6: 309.

51. Ahmed S, Ahmad M, Swami BL, Ikram S (2015) A Review on Plants extract mediated synthesis of silver nanoparticles for antimicrobial applications: A green expertise. Journal of Advanced Research. doi: 10.1016/j.jare.2015.02.007

52. Ahmed S, Ahmad M, Swami BL, Ikram S (2015) Green synthesis of silver nanoparticles using Azadirachta indica aqueous leaf extract. Journal of Radiation Research and Applied Science, 10.1016/j.jrras.2015.06.006

53. Madhumathi K, Sudheesh Kumar PT, Abilash S, Sreeja V, Tamura $H$ Manzoor K, Nair SV, Jayakumar R (2010) Development of novel chitin/ nanosilver composite scaffolds for wound dressing applications. Journal of Materials Science: Materials in Medicine 21: 807-813.

54. Chen M, Yang Z, Wu H, Pan X, Xie X, Wu C (2011) Antimicrobial activity and the mechanism of silver nanoparticle thermosensitive gel. International Journal of Nanomedicine 6: 2873-2877

55. Ana MDP, Angel LDV (2015) Wound healing bionanocomposites based on castor oil polymeric films reinforced with chitosan-modified $\mathrm{ZnO}$ nanoparticles. Biomacromolecules doi: 10.1021/acs.biomac.5b00447 
Citation: Ahmed S, Ahmad M, Jayachandran M, Qureshi MA, Ikram S et al. (2015) Chitosan Based Dressings for Wound Care. Immunochem Immunopathol 1: 106. doi: 10.4172/2469-9756.1000106

56. Qureshi MA, Khatoon F (2014) Development of Citric Acid Cross Linked Poly (vinyl alcohol) Hydrogel Film, Its Degradability and Effect of Temperature, $\mathrm{pH}$. Advance Science Letter 20: 1414-1419.

57. Qureshi MA, Khatoon $\mathrm{F}$ (2015) In vitro study of temperature and $\mathrm{pH}$ responsive Gentamycin sulphate loaded chitosan based hydrogel film for wound dressing applications. Polymer Plastics Technology \& Engineering 54: 573-580.

58. Qureshi MA, Khatoon F (2015) Open Environment Degradability Study of CS/ PVP/PNIPAm Hydrogel Film. Journal of Applicable Chemistry 4: 903-908.

59. Qureshi MA, Khatoon F, Ahmed S (2015) An overview on wounds, their issues and natural remedies for wound healing. Biochemistry \& Physiology 4: http: // dx.doi.org/10.4172/2168-9652.1000165

60. Silva SS, Popa EG, Gomes ME, Cerqueira M, Marques AP, et al. (2013) An investigation of the potential application of chitosan/aloe-based membranes for regenerative medicine. Acta Biomaterelia http: //dx.doi.org/10.1016/j. actbio.2013.02.027

61. Tanodekaew S, Prasitsilp M, Swasdison S, Thavornyutikarn B, Pothsree T, et al. (2004) Preparation of acrylic grafted chitin for wound dressing application Biomaterials 25: $1453-1460$.
62. Ribeiro MP, Espiga A, Silva D, Baptista P, Henriques J, et al. (2009) Development of a new chitosan hydrogel for wound dressing. Wound Repair Regeneration 17: 817-824

63. Zhang Z, Yang D, Nie J (2008) Chitosan/polyethylene glycol diacrylate films as potential wound dressing material. International Journal of Biological Macromolecule 43: 456-462.

64. Mi FL, Shyu SS, Chen CT, Schoung JY (1999) Porous chitosan microspheres suitable for controlling the antigen release of Newcastle disease vaccine: Preparation of antigen-adsorbed microsphere and in vitro release. Biomaterials 20: $1603-1612$.

65. Liu X, Ma L, Mao Z, Gao C (2011) Chitosan-Based Biomaterials for Tissue Repair and Regeneration. Advance Polymer Science 244: 81-128.

66. Tangpasuthadol V, Pongchaisirikul N, Hoven VP (2003) Surface modification of chitosan films. Effects of hydrophobicity on protein adsorption. Carbohydrate Research 338: 937-942.

67. Gonzalez JS, Maiolo AS, Ponce AG, Alvarez VA (2011) Composites based on poly(vinyl alcohol) hydrogels for wound dressing. The Argentine Congress of Bioengineering and Clinical Engineering Conference VII, SABI: 1-4.

68. Kweon DK, Song SB, Park YY (2003) Preparation of water-soluble chitosan/ heparin complex and its application as wound healing accelerator. Biomaterials 24: $1595-1601$. 